\title{
Strain Induced Vortex Core Switching in Planar Magnetostrictive Nanostructures
}

\author{
T. A. Ostler, ${ }^{1}$ R. Cuadrado, ${ }^{1}$ R. W. Chantrell, ${ }^{1}$ A. W. Rushforth,${ }^{2}$ and S. A. Cavill ${ }^{1,3, *}$ \\ ${ }^{1}$ Department of Physics, The University of York, York YO10 5DD, United Kingdom \\ ${ }^{2}$ School of Physics and Astronomy, The University of Nottingham, Nottingham NG7 2RD, United Kingdom \\ ${ }^{3}$ Diamond Light Source, Harwell Science and Innovation Campus, Chilton, Didcot OX11 ODE, United Kingdom
}

(Received 26 February 2015; published 7 August 2015)

\begin{abstract}
The dynamics of magnetic vortex cores is of great interest because the gyrotropic mode has applications in spin torque driven magnetic microwave oscillators, and also provides a means to flip the direction of the core for use in magnetic storage devices. Here, we propose a new means of stimulating magnetization reversal of the vortex core by applying a time-varying strain gradient to planar structures of the magnetostrictive material $\mathrm{Fe}_{\mathbf{8 1}} \mathrm{Ga}_{\mathbf{1 9}}$ (Galfenol), coupled to an underlying piezoelectric layer. Using micromagnetic simulations we have shown that the vortex core state can be deterministically reversed by electric field control of the time-dependent strain-induced anisotropy.
\end{abstract}

PACS numbers: 75.78.-n, 75.60.Jk, 77.55.Nv, 85.80.Jm

Understanding and controlling magnetism in laterally confined nanostructures has been an area of high interest in the past decade. In planar structures the lateral confinement often leads to a ground state where a vortex core is present, the Landau flux closure and vortex domain configurations being the most obvious examples. Apart from fundamental interest in these topological entities, magnetic structures containing vortex cores have been recognized for applications in magnetic random access memory [1] and microwave oscillators including spin torque vortex oscillators [2]. Excitation of vortex core motion [3-5] is not only essential for microwave emission in spin torque vortex oscillators, but has recently been shown to provide a means to flip the direction of the vortex core itself [3], allowing for data writing in magnetic memory applications.

Magnetization dynamics in general can be stimulated in a number of ways including using time-varying magnetic fields [3], femtosecond laser excitation [6,7], spin injection [8], and even electric fields [9] or strain [10,11]. The latter examples have received renewed attention in response to the renaissance in magnetoelectric multiferroics [12]. Crucially, the ability to control magnetic order with electric fields has the potential to vastly reduce the energy requirements compared to magnetic field or spin polarized current control. At quasistatic time scales the manipulation of magnetic domains by the application of strain in the soft magnetostrictive material Galfenol $\left(\mathrm{Fe}_{\mathbf{8 1}} \mathrm{Ga}_{19}\right)$ has been reported in the recent works of Lou [13], Parkes [14], and Cavill [15]. In these studies of composite magnetoelectrics, a voltage induced strain, from a piezoelectric (PE) or ferroelectric substrate, acts as an additional uniaxial anisotropy, modifying the magnetic free energy and

Published by the American Physical Society under the terms of the Creative Commons Attribution 3.0 License. Further distribution of this work must maintain attribution to the author(s) and the published article's title, journal citation, and DOI. allowing reorientation of the magnetic easy axis. The application of voltage-induced strain to these materials has also been shown to significantly tune the high frequency precessional motion of ferromagnetic resonance (FMR) in both thin films [16] and planar microstructures displaying a uniform magnetization and vortex core [10], respectively. While the study of static strain-induced modification of magnetic domain patterns has received considerable attention, the interaction of a dynamic strain with magnetism in thin films and microstructures has lagged behind. Recent exceptions are the works of Jäeger et al. [17] and Cherepov et al. [18], who demonstrate coherent high-amplitude gigahertz precession of the magnetization in $\mathrm{Fe}_{81} \mathrm{Ga}_{19}$ and $\mathrm{Ni}$ thin films, respectively, after excitation by high frequency strain waves. Excitation of the magnetization dynamics as a result of high frequency inverse magnetostriction opens up the possibility of ultrafast acoustic control of magnetic devices.

In this Letter we present a new method for stimulating fast magnetization dynamics in magnetic vortex core structures by utilizing strain coupling in a magnetostrictive FM-PE multilayer. By inducing a time-varying strain, via the application of a time-varying electric field applied to the PE layer, we show that vortex core oscillations can be induced in systems where the symmetry of the flux closure state is broken and is capable of reversing the vortex core.

The magnetic system considered in the present study is based upon a composite magnetoelectric system consisting of a hybrid magnetostrictive-PE multilayer. This type of multilayer allows for the voltage-induced strain to be coupled effectively to the magnetization. For simplicity we assume that the frequency response of the PE layer is flat across the frequency range of interest and provides a strain magnitude of $\approx 10^{-4}$ to the magnetostrictive layer as reported in Ref. [15]. The micromagnetic model was constructed using the Landau-Lifshitz-Bloch equation of motion for magnetic macrospins $[19,20]$. The parameters 
used are based on typical values found in the literature for an epitaxial thin film of the magnetostrictive material $\mathrm{Fe}_{\mathbf{8 1}} \mathrm{Ga}_{\mathbf{1 9}}$ grown on $\operatorname{GaAs}(001)$, namely, $K_{1}^{c}(100)=32.7 \mathrm{~kJ} \mathrm{~m}^{-3}$, $K_{1}^{u}(110)=8.6 \mathrm{~kJ} \mathrm{~m}^{-3}, M_{s}=1.378 \mathrm{MJ} \mathrm{T}^{-1} \mathrm{~m}^{-3}$, and $A=$ $1.3 \times 10^{-11} \mathrm{~J} \mathrm{~m}^{-1}$. Details of the model can be found in Sec. 1 of the Supplemental Material [21].

It is well known that the application of static uniaxial strain to a magnetostrictive material induces an additional uniaxial anisotropy [15] to the magnetic free energy. The uniaxial strain-induced anisotropy can be written

$$
E_{\text {strain }}=-\frac{3}{2} \varepsilon_{y} \lambda_{010}\left(c_{21}-c_{11}\right)\left(\boldsymbol{m} \cdot \hat{\boldsymbol{n}}_{\boldsymbol{s}}\right)^{2},
$$

where $\hat{\boldsymbol{n}}$ is the direction of the strain, assumed in this work to be along the $y$ [010] direction. The factor $\frac{3}{2} \lambda_{010}\left(c_{21}-c_{11}\right)$ is the magnetoelastic constant, which has a value of $\approx 16 \mathrm{MJ} \mathrm{m}^{-3}$ consistent with Ref. [16], and the tensile strain in the $y$ direction, $\varepsilon_{y}$, was taken (unless otherwise stated) to be $2.5 \times 10^{-4}$. The modulation of the strain by applying an oscillating electric field to the PE layer leads to a time-dependent form of Eq. (2):

$$
E_{\text {strain }}=-\frac{3}{2} \varepsilon_{y} \lambda_{010}\left(c_{21}-c_{11}\right)\left(\boldsymbol{m} \cdot \hat{\boldsymbol{n}}_{\boldsymbol{s}}\right)^{2} \cos \left(\omega_{\mathrm{PE}} t\right)
$$

where $\omega_{\mathrm{PE}}$ is the frequency of the electric field induced strain.

We begin by investigating the effects of the PE layer excitation frequency $f_{\mathrm{PE}}$ on the magnetization dynamics of a uniform monodomain state typically found when in an applied magnetic bias field. In our simulations, a spatially uniform time-varying strain is applied along the [010] crystallographic direction at frequency $f_{\mathrm{PE}}\left(\omega_{\mathrm{PE}}=2 \pi f_{\mathrm{PE}}\right)$ with the bias field along the [100] direction (the driving field is initially perpendicular to the bias field). To quantify the effect of the time-varying strain on the magnetization we measure the rms deviation in the $z$ component of the magnetization $\left(m_{z}\right)$, which is effectively the precessional cone angle, as this is largest at resonance.
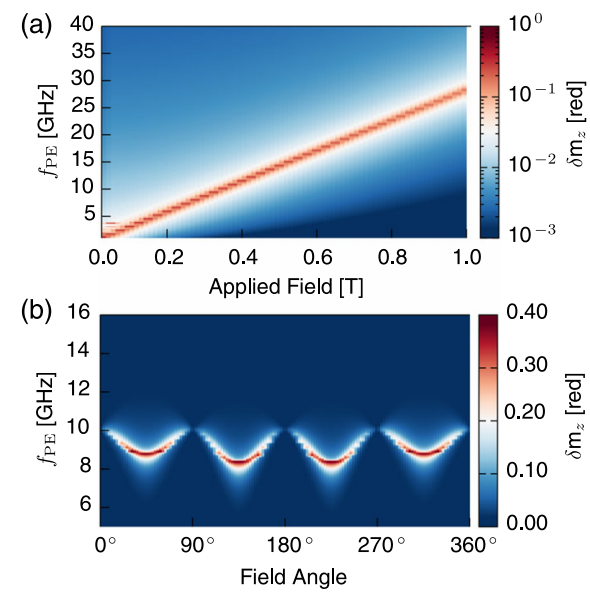

FIG. 1 (color online). Average magnetization fluctuation $\delta m_{z}$ as a function of the driving frequency of the piezoelectric layer and applied field. (a) Bias field along [100] and (b) bias field $=0.3 \mathrm{~T}$.
Figure 1(a) shows the variation of the rms deviation in $m_{z}\left(\delta m_{z}\right)$ as a function of $f_{\mathrm{PE}}$ and fixed applied bias field $B$. With increasing bias field there is a linear increase in the resonance frequency of the system, consistent with standard microwave driven ferromagnetic resonance. As the bias field increases, $\delta m_{z}$ at resonance decreases in magnitude due to the reduction in the precessional cone angle [28]. In addition, as the in-plane bias field increases in strength the cone of precession becomes more elliptical, further emphasizing the reduction in $\delta m_{z}$. Figure 1(b) shows how the resonance frequency varies with the angle of the applied field $(0.3 \mathrm{~T})$ from the [100] direction. The angular dependence of the resonance reflects the magnetocrystalline anisotropies present in the $\mathrm{Fe}_{\mathbf{8 1}} \mathrm{Ga}_{\mathbf{1 9}}$ film, namely, a dominant cubic anisotropy along the [100/010] directions in addition to a weaker uniaxial anisotropy, typical for Fe compounds grown epitaxially on GaAs [11,13,15-17], along the [110] direction. As can be seen in Fig. 1(b) the maximum in $\delta m_{z}$ occurs when the magnetic bias field points along a magnetic hard axis, i.e., the $[110 / \overline{1} 10 / 1 \overline{1} 0 / \overline{1} \overline{1} 0]$ i.e., the directions. This is predominantly due to the relative ease of reorientating a magnetic moment away from a hard axis compared to moving it from an easy axis. As such, one would intuitively expect that for a given excitation the amplitude of precession is greater when the moment is initially oriented along a hard axis. As evidenced by Fig. 1(a), a time-varying sinusoidal strain of the appropriate frequency to meet the FMR conditions excites the uniform precessional mode.

We next demonstrate the effect of a time-varying strain on the magnetization dynamics of a planar nanostructure containing a flux closed domain structure with a central vortex core. In order to calculate the spatially resolved magnetization dynamics we extend our numerical model to simulate a square planar structure of dimensions $500 \times 500 \times 10 \mathrm{~nm}$ with a rectangular cell size of $2 \times 2 \times 10 \mathrm{~nm}$. For the following we also include the exchange interaction between the macrospins and the long ranged demagnetization fields. The value of the Gilbert damping constant used in the simulations is 0.02 , similar to the value (0.017) that has been measured previously in $\mathrm{Fe}_{\mathbf{8 1}} \mathrm{Ga}_{\mathbf{1 9}}$ thin films [16]. At equilibrium the core resides in the center of the film, minimizing the competing energy terms. In the following, we have studied the size of the displacement of the vortex core from its equilibrium (the orbit). As the damping parameter used in the simulations is slightly larger than that observed experimentally, we expect that our results would underestimate the vortex core displacement due to an exponential decrease of the vortex core displacement with increasing damping, see Sec. 2 of the Supplemental Material [21].

For efficient generation of the ground state vortex configuration we have used the OOMMF simulation package [29] to generate the vortex core structure, which has a diameter of $\approx 7 \mathrm{~nm}$. The vortex core configuration is read into our Landau-Lifshitz-Bloch program and driven via the straininduced anisotropy in the megahertz regime, at the core gyrotropic frequency [10], to excite the oscillations of the 
core state around its equilibrium. The first point to note here is that the excitation of the magnetization dynamics via a time-dependent strain-induced anisotropy has some key differences with the time-varying applied field case. When a spatially uniform time-varying field is applied to the Landau flux closure state, moments in each domain rotate to align with the field direction in addition to a displacement of the vortex core. However, when a spatially uniform time-varying uniaxial strain-induced anisotropy is applied, each domain aligns more strongly along the axis of the anisotropy. Thus, due to the symmetry of the Landau flux closure state there is (almost) no motion of the vortex core as there are equal numbers of macrospins whose energy is minimized by aligning with the strain induced uniaxial anisotropy direction.

This is shown schematically in Fig. 2(a). To break this symmetry we introduce a linear gradient in the magnitude of the strain-induced anisotropy of the form $\varepsilon(y)=$ $(d \varepsilon / d y) y$ and the system is driven around the frequency of the vortex gyrotropic mode, which, for the material parameters we have used, is $\sim 290 \mathrm{MHz}$. Strain gradients could be realized experimentally by patterning the PE layer and electrodes [30] or by forming a wedged dielectric between the PE layer and the metallic contact.

The dynamics of the magnetization are shown in Fig. 2(b). The deviation of the vortex core from its unperturbed position grows with time until the amplitude of the oscillation equilibrates to a constant value. We

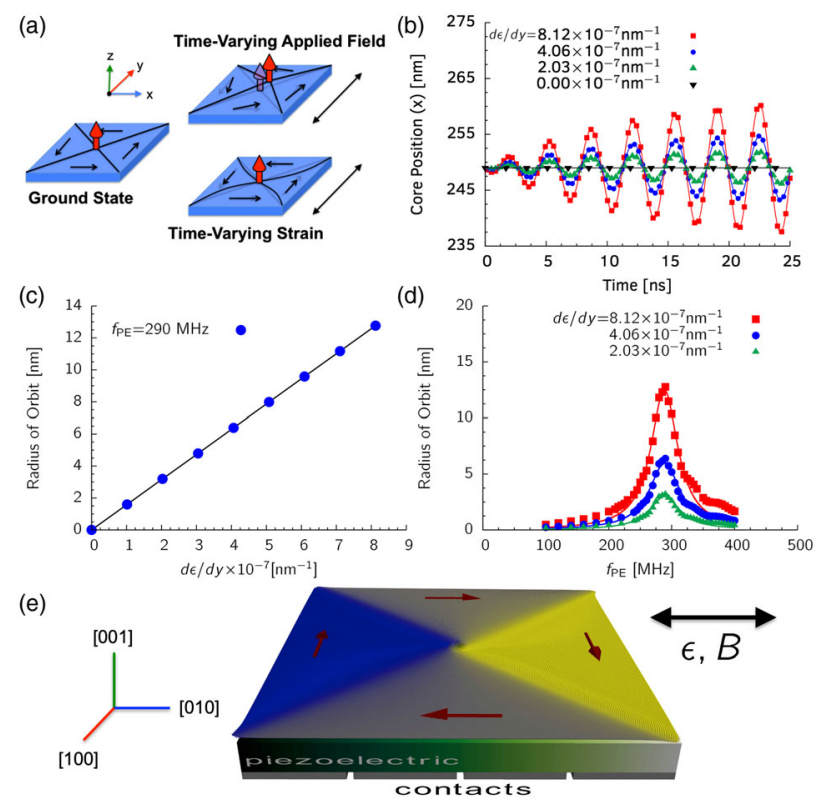

FIG. 2 (color online). The effect of a time and spatially varying strain on the dynamics of a magnetic vortex core. (a) Differences between using a time-varying magnetic field (upper right figure) and the case of no spatial gradient in the time-varying straininduced anisotropy (lower right figure). (b) Dynamics of the $x$ position of the vortex core as a function of time and strain gradient during the first $25 \mathrm{~ns}$. (c) Steady state radius of the oscillations as a function of strain gradient. (d) Steady state radius of the orbit of the vortex core as a function of the driving frequency of the PE. (e) Schematic of the device concept. determine the core position by fitting the time-resolved data and extracting the amplitude of the motion. Figure 2(c) shows the effect of the strain gradient on the size of the steady state vortex core motion around equilibrium when driven at the core gyrotropic frequency of $290 \mathrm{MHz}$. The amplitude of the vortex core motion clearly shows a linear increase with strain gradient, reaching an orbital diameter of $30 \mathrm{~nm}$ for $d \varepsilon / d y \sim 9 \times 10^{-7} \mathrm{~nm}^{-1}$. Figure 2(d) shows the steady state radius of the orbit as a function of the frequency of the strain induced by the PE layer. As the drive frequency is swept towards the natural gyrotropic frequency of the core, the motion increases in amplitude and is maximized at resonance. As expected, the linewidth of the gyrotropic mode driven by the time-varying strain is the same as for the field driven case, see the Supplemental Material [21]. Finally we note that replacing the square structure with a circular one, typically used to generate vortex states, yields the same result (see the Supplemental Material [21]), demonstrating that the shape of the structure is not critical to the physics of strain driven gyrotropic motion.

While very little is known about the effects of timevarying strain on the dynamics of this kind of planar magnetostrictive structure, there is a large body of literature investigating the influence of applied magnetic fields [8] with or without static strain [16]. To that end we have studied the interaction of the time-varying strain-induced anisotropy and a time-varying Zeeman field both applied in the $y$ direction. By applying a time-varying Zeeman field of
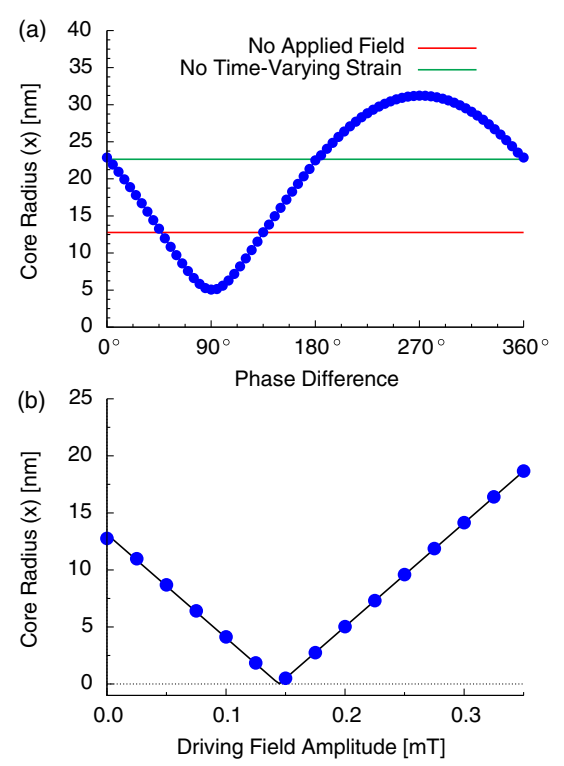

FIG. 3 (color online). The result of a combination of a timevarying Zeeman field and time and spatially varying strain gradient. (a) The maximum vortex core displacement, with the inclusion of the time-varying Zeeman term of amplitude $0.2 \mathrm{mT}$, occurs at $270^{\circ}$. (b) The effect of the magnitude of the timevarying Zeeman field when the phase shift between the two driving terms is $+90^{\circ}$. The black solid line represents a fit to the modulus of a linear line and the point at which it goes to zero represents a balancing of the Zeeman and time-varying strain anisotropies, occurring at around $0.14 \mathrm{mT}$. 


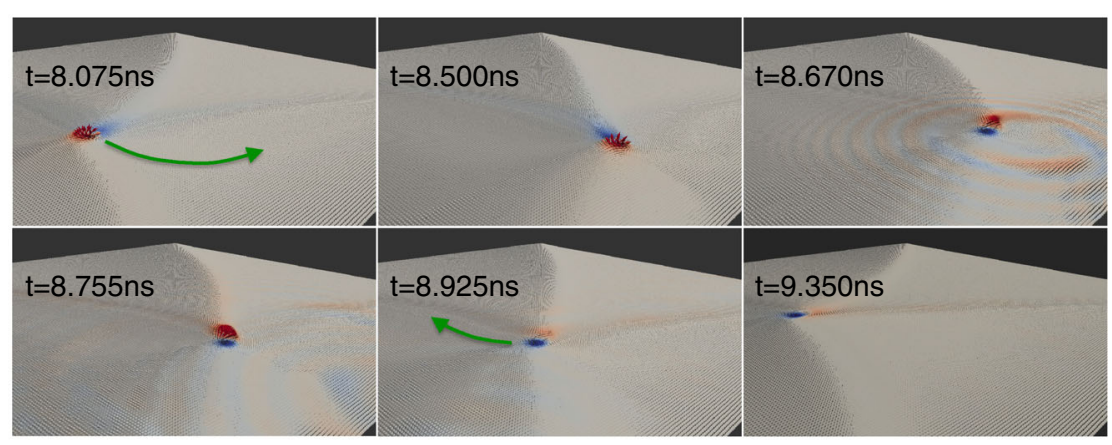

FIG. 4 (color online). Snapshots of the vortex core motion during switching by a time-varying strain. At $8.075 \mathrm{~ns}$ the vortex core motion is counterclockwise as indicated by the green arrow. At 8.670 ns the antivortex-vortex pair, which has arisen due to the driving force, annihilates with the expected ejection of spin waves from the core. After the core switches the motion of the vortex is in the opposite direction and the switching is complete. The color of the macrospins corresponds to the $z$ component of the magnetization. The full time sequence is available in the Supplemental Material [21].

$0.2 \mathrm{mT}$ with a given phase between the time-varying straininduced anisotropy, we can reduce or enhance the motion of the core by simply adjusting the phase between the timevarying $E$ field (strain) and $B$ field (Zeeman).

Figure 3(a) shows the calculated vortex core displacement as a function of the phase between the time-varying strain-induced anisotropy and time-varying Zeeman field. The results demonstrate that the core oscillations can be almost completely suppressed by a $90^{\circ}$ phase shift. At this phase the Zeeman term causes the core to move in the opposite rotational sense to that caused by the strain term alone, suppressing the motion. On the other hand, the core oscillations can be enhanced for a phase shift of $270^{\circ}$, which is when the two terms drive the core in the same rotational sense. By adjusting the magnitude of the driving Zeeman field (with the two driving terms $90^{\circ}$ out of phase) the core motion can be completely suppressed [Fig. 3(b)], in our case with a field of around $0.14 \mathrm{mT}$.

Thus far we have demonstrated that a time-varying strain field can be utilized to induce magnetization dynamics at various resonances. However, for our mechanism to be used in any potential device, particularly for realizing low energy storage technology, the possibility to reverse the magnetic order parameter must be realized. By further increasing the magnitude of the time-varying strain gradient across the device we can deterministically switch the vortex core without the need for applied magnetic fields or spin currents.

Figure 4 shows a sequence of snapshots of the macrospins after the time-varying strain starts to drive the vortex oscillations. Initially, the core moves counterclockwise (top left panel), as indicated by the green arrow. At 8 ns the position of the core is already quite far from its central equilibrium. Once the core motion reaches a critical velocity of $306 \mathrm{~m} \mathrm{~s}^{-1}$ [4], an antivortex-vortex pair nucleates. The antivortex annihilates with the original vortex, as shown in the top right panel, resulting in the ejection of high frequency spin waves, and only the new vortex, with an opposite polarization, remains in the structure.

Finally, the motion of the reversed vortex core begins to build again, but now moving in a clockwise direction. The full time sequence can be found in the Supplemental Material [21].

For this system we find that a threshold gradient in the strain of $2.03 \times 10^{-5} \mathrm{~nm}^{-1}$, driven at resonance
(290 MHz), is required to reverse the vortex core with an energy cost of $<1 \mathrm{pJ}$ [31]. This is considerably smaller than for the field driven case studied, which is $>0.1 \mathrm{~nJ}$ for the same system [32]. By carefully selecting the material properties for a particular device size the magnetoelastic and shape anisotropy could be finely balanced, resulting in a lower threshold strain gradient, and therefore energy, needed to switch the vortex core.

Conclusions. - In this Letter we have constructed a micromagnetic model of the magnetostrictive material Galfenol, based on the Landau-Lifshitz-Bloch equation of motion. We have proposed a means by which the magnetization dynamics can be manipulated through time-induced strain in a composite multiferroic consisting of a piezoelectric coupled to a soft magnetostrictive layer. For single domain thin films we have shown that a timevarying strain-induced anisotropy in the gigahertz regime can drive the system to the uniform FMR mode.

By driving the dynamics in Galfenol in the megahertz regime by the use of a time-varying strain we have demonstrated that in a Landau flux closure state it is not possible to drive the motion of the core due to symmetry considerations. By introducing a gradient in the strain the spatial symmetry can be broken, resulting in a motion of the core that scales linearly with the magnitude of the gradient. By growing magnetic materials with large magnetoelastic energies directly onto ferroelectric substrates, such as $\mathrm{Ni}$ on $\left[\mathrm{Pb}\left(\mathrm{Mg}_{1 / 3} \mathrm{Nb}_{2 / 3}\right) \mathrm{O}_{3}\right]_{(1-x)}{ }^{-}\left[\mathrm{PbTiO}_{3}\right]_{x}$, the radius of the core orbit could be significantly increased due to the larger strain. Finally, by increasing the gradient of the strain further we have shown that the vortex core can be reversed using electric field control only. We remark here that it should also be possible to drive vortex core dynamics in structures that have a different symmetry than the Landau flux closure state.

Our findings demonstrate a new means of inducing magnetization dynamics in complex planar magnetic structures such as vortex cores. We have shown that it is possible to reverse the core polarity without the need of a Zeeman field, which will be of potential importance in devices such as spin-torque nano-oscillators or magnetic random access memory.

We gratefully acknowledge the funding from the EC's FP7 Program (FP7/2007-2013) under Grant Agreement 
No. 281043 FemtoSpin, EPSRC under EP/K000225/1 and $\mathrm{EP} / \mathrm{K} 031589 / 1$, as well as funding from Seagate Technology and Diamond Light Source. A. W. R. acknowledges support from EPSRC Career Acceleration Fellowship EP/H003487/1.

*Corresponding author. stuart.cavill@york.ac.uk

[1] R. Hertel, Nat. Nanotechnol. 8, 318 (2013).

[2] V. Pribiag, I. N. Krivorotov, G. D. Fuchs, P. M. Braganca, O. Ozatay, J. C. Sankey, D. C. Ralph, and R. A. Buhrman, Nat. Phys. 3, 498 (2007).

[3] B. Van Waeyenberge et al., Nature (London) 444, 461 (2006).

[4] K. Lee, S.-K. Kim, Y.-S. Yu, Y.-S. Choi, K. Yu. Guslienko, H. Jung, and P. Fischer, Phys. Rev. Lett. 101, 267206 (2008).

[5] R. Hertel, S. Gliga, M. Fähnle, and C. M. Schneider, Phys. Rev. Lett. 98, 117201 (2007).

[6] T. A. Ostler et al., Nat. Commun. 3, 666 (2012).

[7] I. Radu et al., Nature (London) 472, 205 (2011).

[8] K. Ando, S. Takahashi, J. Ieda, H. Kurebayashi, T. Trypiniotis, C. H. W. Barnes, S. Maekawa, and E. Saitoh, Nat. Mater. 10, 655 (2011).

[9] W. Zhu, D. Xiao, Y. Liu, S. J. Gon, and C. Duan, Sci. Rep. 4, 4117 (2014).

[10] D. E. Parkes, R. Beardsley, S. Bowe, I. Isakov, P. A. Warburton, K. W. Edmonds, R. P. Campion, B. L. Gallagher, A. W. Rushforth, and S. A. Cavill, Appl. Phys. Lett. 105, 062405 (2014).

[11] N. Lei et al., Nat. Commun. 4, 1378 (2013).

[12] N. Hur, S. Park, P. A. Sharma, J. S. Ahn, S. Guha, and S.-W. Cheong, Nature (London) 429, 392 (2004).

[13] J. Lou, M. Liu, D. Reed, Y. Ren, and N. X. Sun, Adv. Mater. 21, 2536 (2009).

[14] D. E. Parkes, S. A. Cavill, A. T. Hindmarch, P. Wadley, F. McGee, C. R. Staddon, K. W. Edmonds, R. P. Campion, B. L. Gallagher, and A. W. Rushforth, Appl. Phys. Lett. 101, 072402 (2012).

[15] S. A. Cavill, D. E. Parkes, J. Miguel, S. S. Dhesi, K. W. Edmonds, R. P. Campion, and A. W. Rushforth, Appl. Phys. Lett. 102, 032405 (2013).

[16] D. E. Parkes et al., Sci. Rep. 3, 2220 (2013).

[17] J. V. Jäger et al., Appl. Phys. Lett. 103, 032409 (2013).
[18] S. Cherepov, P. K. Amiri, J. G. Alzate, K. Wong, M. Lewis, P. Upadhyaya, J. Nath, M. Bao, A. Bur, T. Wu, G. P. Carman, A. Khitun, and K. L. Wang, Appl. Phys. Lett. 104, 082403 (2014).

[19] D. A. Garanin, Phys. Rev. B 55, 3050 (1997).

[20] T. A. Ostler, M. O. A. Ellis, D. Hinzke, and U. Nowak, Phys. Rev. B 90, 094402 (2014).

[21] See Supplemental Material at http://link.aps.org/ supplemental/10.1103/PhysRevLett.115.067202 which includes the equivalence of square and circular structures. Supplemental Material contains Refs. [22-27].

[22] U. Atxitia, T. Ostler, J. Barker, R. F. L. Evans, R. W. Chantrell, and O. Chubykalo-Fesenko, Phys. Rev. B 87, 224417 (2013).

[23] U. Atxitia, O. Chubykalo-Fesenko, N. Kazantseva, D. Hinzke, U. Nowak, and R. W. Chantrell, Appl. Phys. Lett. 91, 232507 (2007).

[24] K. Lebecki and U. Nowak, J. Appl. Phys. 113, 023906 (2013).

[25] K. Vahaplar, A. M. Kalashnikova, A. V. Kimel, D. Hinzke, U. Nowak, R. Chantrell, A. Tsukamoto, A. Itoh, A. Kirilyuk, and Th. Rasing, Phys. Rev. Lett. 103, 117201 (2009).

[26] T. A. Ostler, M. O. A. Ellis, D. Hinzke, and U. Nowak, Phys. Rev. B 90, 094402 (2014).

[27] A. J. Newell, W. Williams, and D. J. Dunlop, J. Geophys. Res. 98, 9551 (1993).

[28] M. Farle, Rep. Prog. Phys. 61, 755 (1998).

[29] M. J. Donahue and D. G. Porter, 1999, http://math.nist.gov/ oommf.

[30] M. Rinaldi, T. G. Bjåstad, L. Løvstakken, K. Kristoffersen, and H. Torp, IEEE Trans. Sonics Ultrason. 57, 38 (2010).

[31] The energy needed to switch the core is calculated following the method of J.-M. Hu, Z. Li, L.-Q. Chen, and C.-W. Nan, Nat. Commun. 2, 553 (2011), and is of the order $E \sim n P_{r} S V$, where $n$ is the number of rf cycles needed to switch the core, $P_{r}$ is the remnant polarization of the ferroelectric, $S$ is the device area, and $V$ is the peak amplitude of the rf voltage.

[32] According to Ref. [4] a 30 Oe linear rf field requires $>10 \mathrm{~ns}$ to switch the vortex core for a Fe based device. For a $600 \mathrm{~nm}$ $50 \Omega$ coplanar waveguide (the waveguide is set slightly larger than the $500 \mathrm{~nm}$ device) $0.143 \mathrm{~W}$ is required to generate this field, see G. B. G Stenning et al., New J. Phys. 17, 013019 (2015). In the $10 \mathrm{~ns}$ needed to switch the core the energy requirement is therefore $\sim 1 \mathrm{~nJ}$. 